\title{
The very low angle detector for high-energy inelastic neutron scattering on the VESUVIO spectrometer
}

\author{
E. Perelli Cippo ${ }^{\text {a }}$, G. Gorini ${ }^{\mathrm{a}}$, M. Tardocchi ${ }^{\mathrm{a}}$, A. Pietropaolo, ${ }^{\mathrm{b}, \mathrm{c}, *}$, \\ C. Andreani ${ }^{\text {, }}$ R. Senesi ${ }^{\mathrm{d}}$, N.J. Rhodes ${ }^{\mathrm{e}}$, E.M. Schooneveld ${ }^{\mathrm{e}}$ \\ ${ }^{a}$ Dipartimento di Fisica "G. Occhialini”, Universitá degli Studi di Milano-Bicocca, Piazza della Scienza 3, 20126 Milano, Italy \\ bDipartimento di Fisica "G. Occhialini”, CNISM-Universitá degli Studi di Milano-Bicocca, Piazza della Scienza 3, 20126 Milano, Italy \\ ${ }^{\mathrm{c}} N A S T$ Center (Nanoscienze-Nanotecnologie-Strumentazione), Universitá degli Studi di Roma Tor Vergata, \\ via della Ricerca Scientifica 1, 00133 Roma, Italy \\ ${ }^{\mathrm{d}}$ Dipartimento di Fisica and Centro NAST (Nanoscienze-Nanotecnologie-Strumentazione), Universitá degli Studi di Roma Tor Vergata, \\ via della Ricerca Scientifica 1, 00133 Roma, Italy \\ ${ }^{\mathrm{e}}$ ISIS Facility, Rutherford Appleton Laboratory, Chilton, Didcot, Oxfordshire 0QX OX11, UK
}

Received 5 February 2008; accepted 11 February 2008

Available online 19 February 2008

\begin{abstract}
The Very Low Angle Detector (VLAD) bank has been installed on the VESUVIO spectrometer at the ISIS spallation neutron source. The new device allows for high-energy inelastic neutron scattering measurements, at energies above $1 \mathrm{eV}$, maintaining the wave vector transfer lower than $10 \AA^{-1}$. This opens a still unexplored region of the kinematical $(q, \omega)$ space, enabling new and challenging experimental investigations in condensed matter. This paper describes the main instrumental features of the VLAD device, including instrument design, detector response, and calibration procedure.
\end{abstract}

(C) 2008 Elsevier B.V. All rights reserved.

Keywords: Neutron scattering; Neutron instrumentation; HINS

\section{Introduction}

The advent of the pulsed neutron sources has opened up new fields of investigation in condensed matter, as the intense fluxes of epithermal neutrons (above $500 \mathrm{meV}$ ) allow to achieve energy, $\omega$, and wave vector, $q$, transfers unaccessible at steady state reactors. The investigation of condensed matter at small scales of time $\left(\leqslant 10^{-15} \mathrm{~s}\right)$ and distances $\left(\leqslant 1 \AA^{-1}\right)$ is very attractive, as information on the single particle dynamics, namely atomic momentum distribution $n(p)$ and mean kinetic energy $\left\langle E_{\mathrm{K}}\right\rangle$, can directly be accessed through the deep inelastic neutron scattering (DINS) technique [1-3]. On the other hand, epithermal neutrons allow to access the kinematical region of the $(q, \omega)$

\footnotetext{
*Corresponding author at: Dipartimento di Fisica "G. Occhialini", CNISM-Universitá degli Studi di Milano-Bicocca, Piazza della Scienza 3, 20126 Milano, Italy. Fax: + 390264482367.

E-mail address: antonino.pietropaolo@mib.infn.it (A. Pietropaolo).
}

space characterized by $\omega \geqslant 1 \mathrm{eV}$ and $q \leqslant 10 \AA^{-1}$, i.e. the high energy inelastic neutron scattering (HINS) regime $[4,5]$. HINS enables experimental studies on the dispersion relations of high energy excitations in metals, semiconductors and insulators, high lying molecular rotationalvibrational states, molecular electronic excitations, and the electronic level in solids [6-9].

The most effective way to fulfill the HINS kinematical conditions is to use the inverse geometry configuration, detecting scattered neutrons well above $1 \mathrm{eV}$ at small angles [10-12]. To this aim, the experimental concept of the Resonance Detector (RD), proposed in the late 70s [13,14], has been revisited and optimized for the VESUVIO beam line $[15,16]$ at the ISIS spallation neutron source [17]. Different types of photon detectors have been successfully tested on VESUVIO in the RD configuration for DINS measurements, at scattering angles above $20^{\circ}$ [18-20]. Their use at the very small scattering angles $\left(\leqslant 5^{\circ}\right)$ required for high $\omega$ and low $q$ was a more recent development 
and required structural modifications of the original spectrometer layout. The beam line of the instrument was modified by tightening the incident neutron collimation, and by fitting a new vacuum tank which provides minimum attenuation of the neutrons scattered at low angles. A prototype of the VLAD bank was previously constructed and successfully tested with benchmark HINS measurements [4].

This paper concerns the main features of the final VLAD device, including instrument design, detector response, and calibration procedure. Section 2 provides a brief overview of the new scientific opportunities opened by this instrument development, while Section 3 discusses the kinematical requirements of HINS on an inverse geometry time of flight spectrometer. Sections 4 and 5 provide a description of the system and its calibration. Some concluding remarks are made in Section 6.

\section{New science requirements}

Accessing the HINS regime will allow for new experimental studies in condensed matter. HINS provides information on the dynamical structure factor $S(\vec{q}, \omega)$ on the nuclear interaction of neutrons with atomic nuclei. $S(\vec{q}, \omega)$ depends on the density of states $g(\omega)$ and on the vibration amplitude $\left\langle u^{2}\right\rangle$ of each atom in the molecule, weighted by its neutron scattering cross-section. In a hydrogen containing sample, the large incoherent crosssections and the low mass of the proton ensure that the contribution to $S(\vec{q}, \omega)$ coming from molecular vibrations involving hydrogen dominates the scattering intensity. In the kinematical region at low wave vector and high energy transfers (HETs), HINS allows access to the one-phonon component, $S_{m, \pm 1}(\vec{q}, \omega)$, of hydrogen-projected scattering function, $S_{m}(\vec{q}, \omega)$. The expression of the dynamical structure factor in this case is given, in the isotropic approximation, by [21]

$$
\begin{aligned}
S_{m, \pm 1}(\vec{q}, \omega)= & \frac{\hbar q^{2}}{4 m} \exp \left(-\frac{1}{3} q^{2}\left\langle u^{2}\right\rangle_{m}\right) \\
& \times \frac{g(\omega)}{\omega}\left[\operatorname{coth}\left(\frac{\omega}{2 k_{\mathrm{B}} T}\right)+1\right]
\end{aligned}
$$

where $g(\omega)$ is the density of states projected on the hydrogen atoms of the unit cell and $\left\langle u^{2}\right\rangle_{m}$ is the nuclear mean squared displacement averaged over the hydrogen atoms of the unit cell.

On the other hand, HINS can be induced by the neutron magnetic interaction (through its magnetic moment) with the electron spin of the unpaired electrons. In such cases the magnetic interaction between neutron and electrons is described by the Hamiltonian:

$H_{\text {int }}=-\overrightarrow{\mu_{\mathrm{n}}} \cdot \vec{B}$

where $\overrightarrow{\mu_{\mathrm{n}}}=-\gamma \mu_{\mathrm{N}} \vec{\sigma}$ is the neutron magnetic moment ( $\vec{\sigma}$ being the Pauli spin operator acting on the neutron wave function, $\mu_{\mathrm{N}}$ the nuclear magneton and $\gamma=1.913$ ) and $\vec{B}$ the magnetic field of all electrons [22-26]. Consider a scattering process in which the system undergoes a transition from an initial state at energy $E_{\mathrm{i}}$ to a final state of energy $E_{\mathrm{f}}$, while the neutron is scattered from an initial state of wave vector $\vec{k}_{0}$ and energy $E_{0}$ to a final state of wave vector $\vec{k}_{1}$ and energy $E_{1}$. In the first Born approximation, the double differential scattering crosssection is given by [25]

$\frac{\mathrm{d}^{2} \sigma}{\mathrm{d} \Omega \mathrm{d} E_{0}}=\frac{k_{1}}{k_{0}}|f(\vec{q})|^{2} \delta\left(E_{\mathrm{i}}-E_{\mathrm{f}}-\omega\right)$

where $\vec{q}=\vec{k}_{0}-\vec{k}_{1}, \omega=E_{0}-E_{1}$ and $f(\vec{q})$ is the magnetic form factor. The latter is proportional to the classical electron radius $r_{0}=e / 4 \pi \varepsilon_{0} m_{e} c^{2}$, so that considering Eq. (3) the double differential scattering cross-section becomes proportional to $r_{0}^{2}$. Thus, as compared to the nuclear scattering cross-sections typical of DINS processes (ranging from few barns as for helium isotopes, to several tens of barns for hydrogen), those for magnetic interactions are at least one order of magnitude smaller.

Extending the energy transfer scale above $800 \mathrm{meV}-1 \mathrm{eV}$ with an associated low value of $q$, would allow a significant advance in the study of crystal field levels in rare earth intermetallic compounds and magnetic materials. For experiments involving still larger excitation energies $(2-6 \mathrm{eV})$, epithermal neutrons can be used as a direct probe of the electronic band structure of materials such as semiconductor or insulators. On the HET spectrometer at ISIS, for example, inter-multiplet transitions with energies up to about $800 \mathrm{meV}$ have been observed in Praseodymium [27] employing monochromatic incident neutrons up to about $1.7 \mathrm{eV}$. In order to observe higher lying transitions (e.g. the ${ }^{3} \mathrm{H}_{4} \rightarrow{ }^{1} \mathrm{G}_{4}$ expected at about $1.2 \mathrm{eV}$ ) higher incident and final neutron energies are required, as briefly clarified in the next section.

\section{Kinematical considerations}

From a kinematical point of view, HINS requires that high energy neutrons in the final state be detected at small scattering angles. This can be argued by Fig. 1, where a contour plot of iso- $q$ loci as a function of final energy and energy transfer $\omega$ is shown for three different scattering angles. Indeed, HINS provides access to a region of the $(q, \omega)$ space which is complementary to that of direct geometry chopper spectrometers as well as to other experimental techniques using different probes (see Fig. 2). As a specific example, the HET direct geometry time of flight spectrometer is optimized for high energy magnetic excitations above $50 \mathrm{meV}$, but being a direct geometry instrument the maximum energy loss is limited to about $2 \mathrm{eV}$.

For an inverse geometry instrument, where the scattered neutron energy is selected, there is no kinematical limit to the energy loss [28] and high $\omega$ can be achieved at low $q$ values. 
a

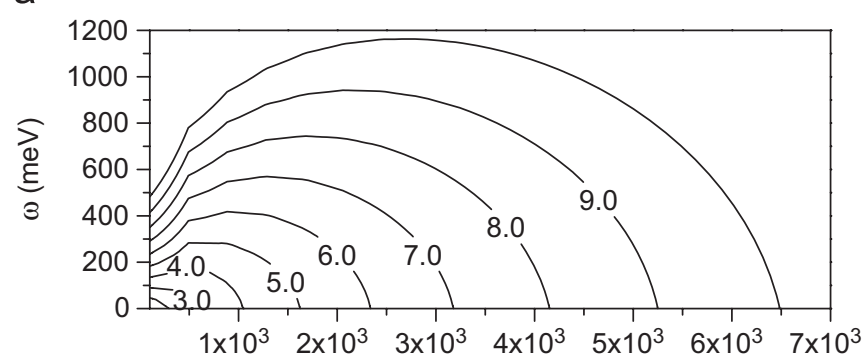

b

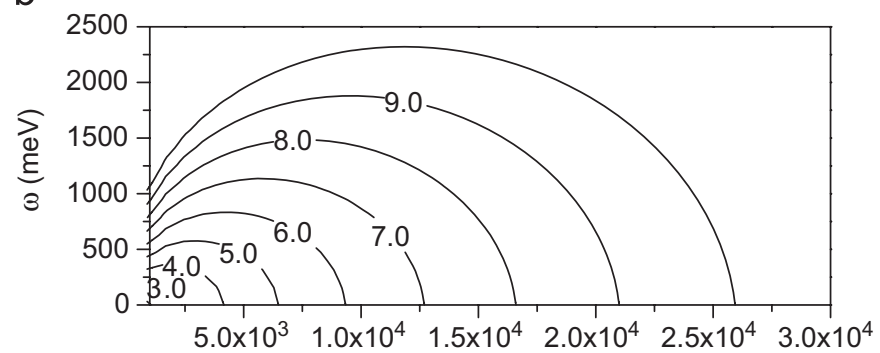

C

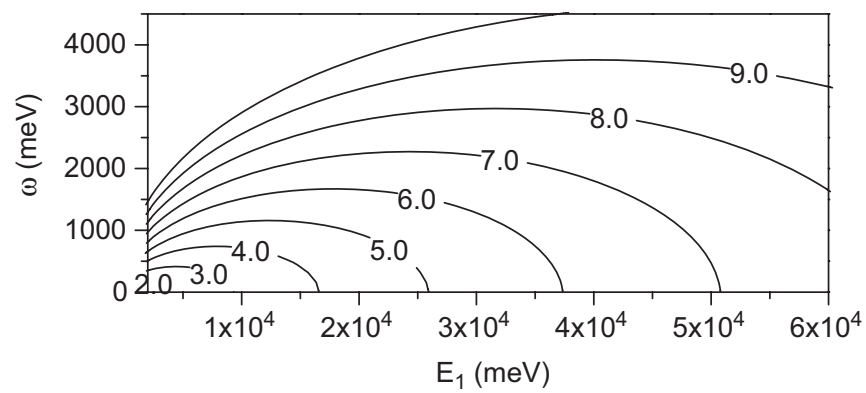

Fig. 1. Contour plots of iso- $q$ loci as a function of final energy $E_{1}$ and energy transfer $\omega$ for three different scattering angles, namely $1^{\circ}$ (a), $3^{\circ}$ (b), and $5^{\circ}(\mathrm{c})$.

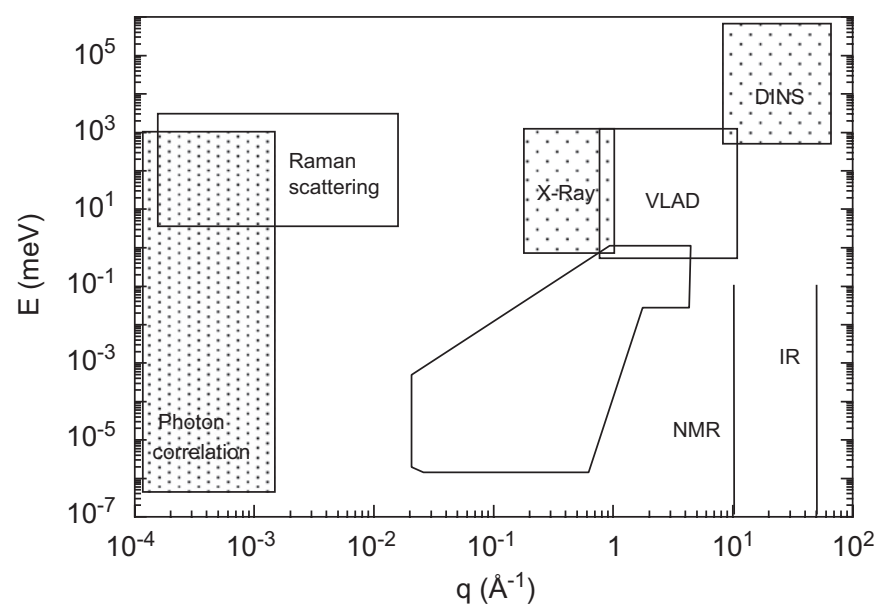

Fig. 2. Schematic representation of the regions of the kinematical $(q, \omega)$ space accessed by different experimental techniques for condensed matter investigation.

\section{Instrument design}

A prototype of the VLAD equipment was mounted on VESUVIO spectrometer in 2003 and was tested through benchmark HINS measurements on a polycrystalline IceIh sample [4]. Those measurements were performed in the RD configuration using four YAP scintillators coupled to

${ }^{238} \mathrm{U}$ analyzers. The use of detectors at small scattering angles required the modification of the VESUVIO spectrometer. In fact, a series of calculations taking into account the size of the neutron spot at the sample position and the beam divergence were performed in order to compute the angular range accessible with the original VESUVIO layout. These calculations indicated that a secondary flight path of $2 \mathrm{~m}$ was necessary in order to avoid the transmitted neutrons to strike the detectors placed at the lowest angle. To reach scattering angles as low as $1^{\circ}$, a reduction of the beam size was achieved by adding a $\mathrm{B}_{4} \mathrm{C}$ insert collimation in the incident beam pipe (see Fig. 3). This provides a 23 $\mathrm{mm}$ penumbra diameter at the sample position and a corresponding improvement of the angular resolution.

Fig. 4 shows a contour plot of iso-angular loci, calculated with a flight path $L_{1}=2 \mathrm{~m}$, as a function of $q$ and $\omega$ and for a final neutron energy of $20 \mathrm{eV}$. The region of the $(q, \omega)$ space accessed is characterized by $q \leqslant 10 \AA^{-1}$ and $\omega$ up to $3 \mathrm{eV}$, for scattering angles in the range $1-6^{\circ}$. As already pointed out in other experimental papers, the effective detection of neutron of energy above $15-20 \mathrm{eV}$ is feasible with the use of the RD configuration. The latter relies upon two main steps: In the first one, the scattered neutron beam impinges onto the analyzer foil which provides the energy analysis by means of $(n, \gamma)$ resonance absorption at a given resonance energy $E_{1}$; in the second step, the prompt gamma-rays are detected to determine the total neutron time of flight. The broad gamma-ray spectrum from the analyzer allows for the use of energy discrimination thresholds that improve the signal to background ratio $(S / B)$, still guaranteeing an acceptable counting efficiency [19].

\section{The VLAD instrumental components and calibration}

The final VLAD bank was installed on VESUVIO in 2005. The new device makes use of a cylindric aluminum vacuum chamber, connected by a bellow to the VESUVIO sample tank and to the transmitted beam pipe. The VLAD chamber is closed by a curved aluminum window reinforced by six radial stems. With this solution the window is only $2 \mathrm{~mm}$ thickness, still maintaining a high vacuum capability. The scattered neutrons travel in vacuum from the sample to the detector, avoiding neutron scattering off air and without intercepting the beam pipe medium. The detector bank is composed of an aluminum frame for detector support and five sets of yttrium-aluminum-perovskite (YAP) detectors, each ring being placed at different scattering angles and flight paths [29].

The YAP crystals (20 in total) have been designed in a trapezoidal form with $6 \mathrm{~mm}$ thickness and effective area of $546 \mathrm{~mm}^{2}$ for the lowest scattering angle and $2150 \mathrm{~mm}^{2}$ for the highest one. A system of aluminum light guides, 


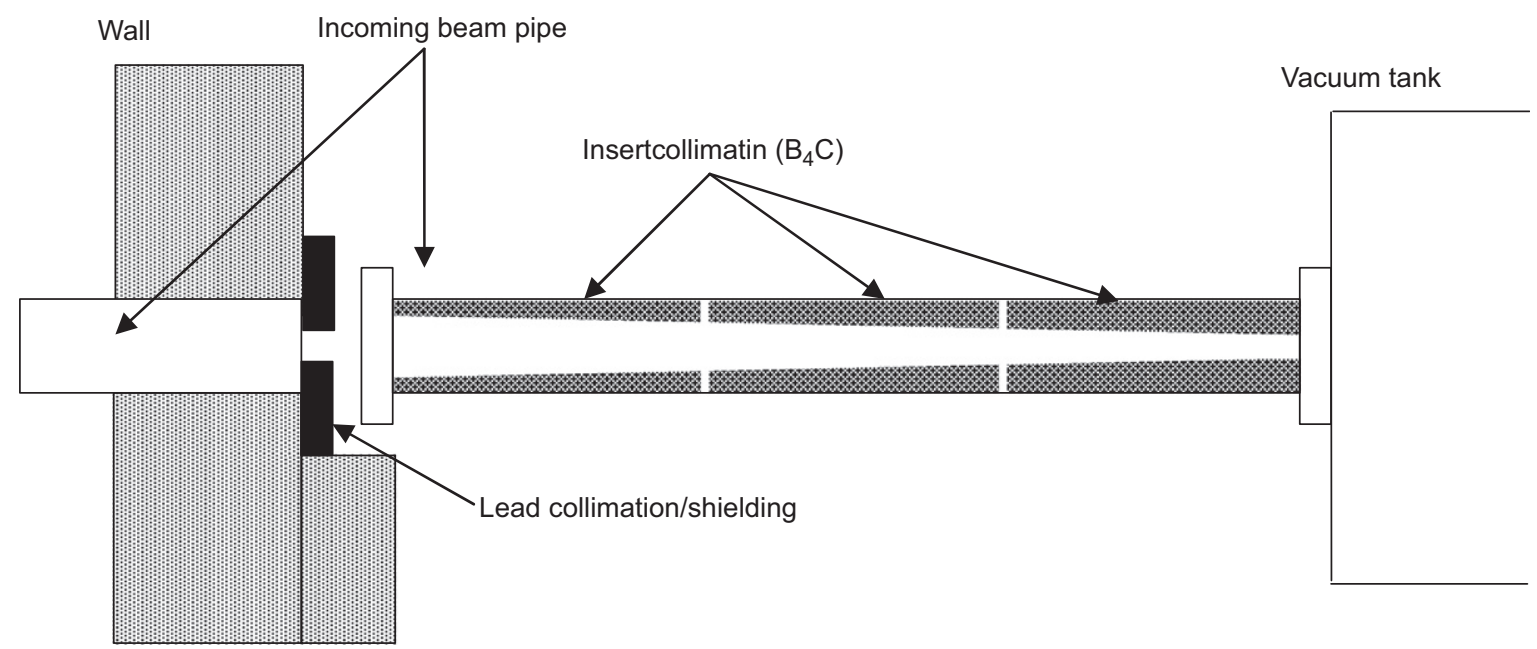

Fig. 3. Schematic drawing of the insert beam collimation designed and realized for the incident beam pipe of the VESUVIO instrument.

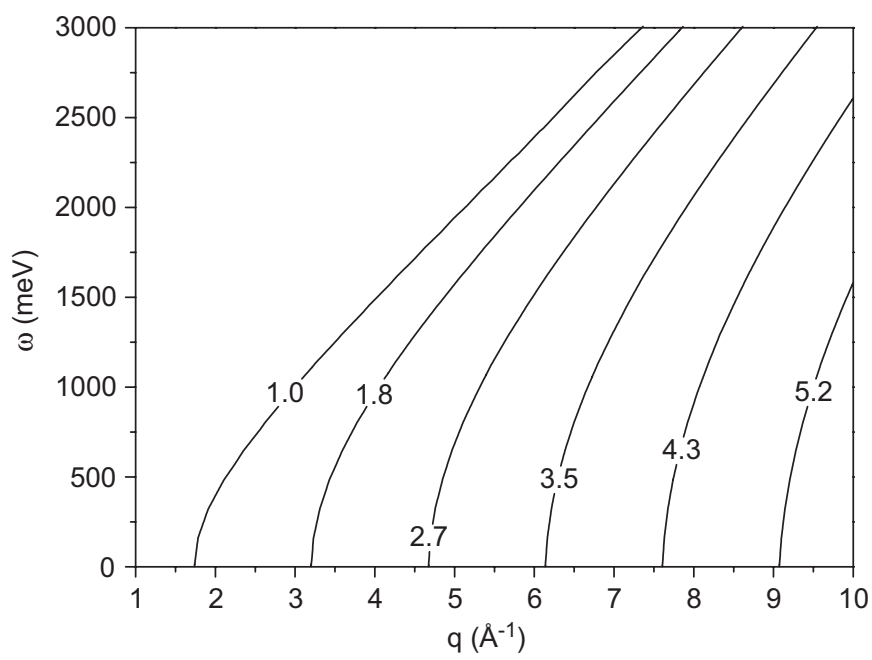

Fig. 4. Region of the $(q, \omega)$ kinematical space accessible on VESUVIO for a final neutron energy of $20 \mathrm{eV}, L_{1}=2 \mathrm{~m}$ and scattering angles below $6^{\circ}$. The values of $q$ and $\omega$ attainable are typical of the HINS regime.

painted inside with diffusing enamel, ensure good reflection for light collection onto the photomultiplier tube.

The performances of the YAP-based detection system was investigated by a series of simulations with GEANT4 code [30]. These simulations aimed to estimate detection efficiency and to study the effect of the cross-talk among detectors and neutron backscattering in the YAP's active medium [31]. The results of the simulations indicated the optimized detectors arrangement for the final VLAD design.

\subsection{Alignment of the detector array}

The vacuum chamber and the VLAD detector assembly are adjustable along the three spatial axes, thus allowing for a precise alignment of the detector array with respect to the neutron beam axis. It was of great importance placing the six detectors of the innermost ring outside the transmitted beam penumbra. To this aim, the relative position of the beam axis and the center of the detector rings were adjusted, taking as a reference the neutron penumbra, determined by means of a neutron-sensitive photographic plate. Then, tests were done to confirm that the detectors were not irradiated by the transmitted beam. In Fig. 6 it can be seen that no resonance peaks from the analyzer foils are visible in the measurement without the scattering sample. A scattering measurement with a plastic sample is also shown for comparison (see also Figs. 5, 6).

\subsection{Flight paths and scattering angles calibration}

The secondary flight path $L_{1}$ and the scattering angle $2 \vartheta$ for each detector in the different rings have been calibrated using HINS data from a $5 \mathrm{~mm}$ thick Perspex slab. From the time positions of the elastic peaks (see lower panel of Fig. 6) the value of $L_{1}$ has been extracted using the relation:

$t=t_{0}+\frac{L_{0}+L_{1}}{v}$.

The time positions of the elastic peaks have been plotted versus $1 / v, v$ being the neutron velocity. A linear fit (see Fig. 7) provided calibrated values of $L_{1}$ and of the time delay $t_{0}$.

The distance $R$ of each detector from the beam pipe axis (aligned with the beam axis) was determined by measuring the diameters of the beam pipe at the different detectors positions. The scattering angle $2 \vartheta_{i}$ for each ring was then calculated through the relation:

$2 \vartheta_{i}=\arcsin \left(\frac{R}{L_{1, i}}\right)$.

$L_{1, i}$ being the secondary flight path relative to the $i$ th ring of the array $(i=1,2, \ldots, 5)$. Table 1 summarizes values of the calibrated instrument parameters. The experimental values agree with the construction values. As the angular positions of the detectors within a given ring are similar, the data acquired by the YAP detectors belonging to the 

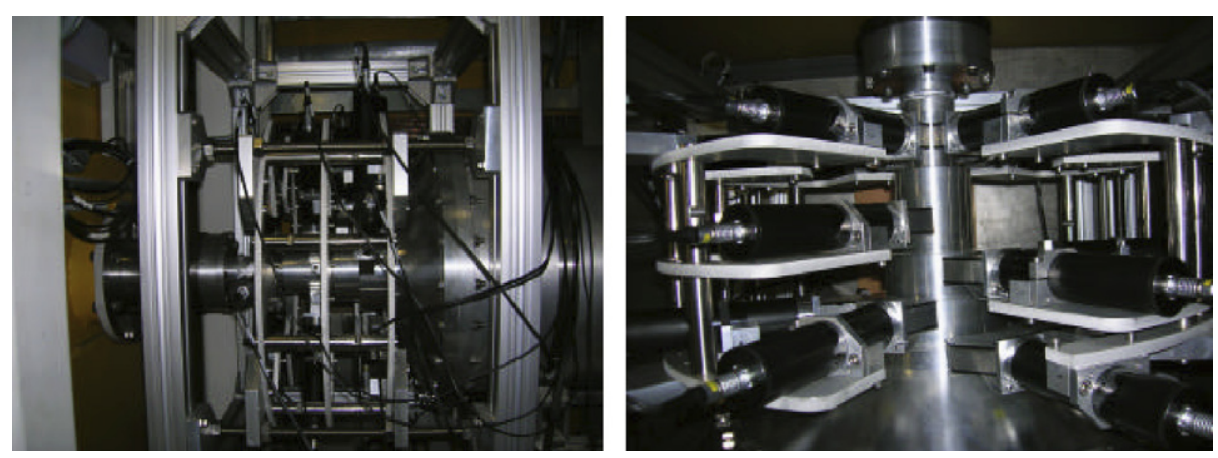

Fig. 5. Pictures showing the VLAD detectors array. The left picture is the lateral view (neutrons coming from the right), while the right one is the top view (neutrons coming from the bottom) of the device, respectively.

a

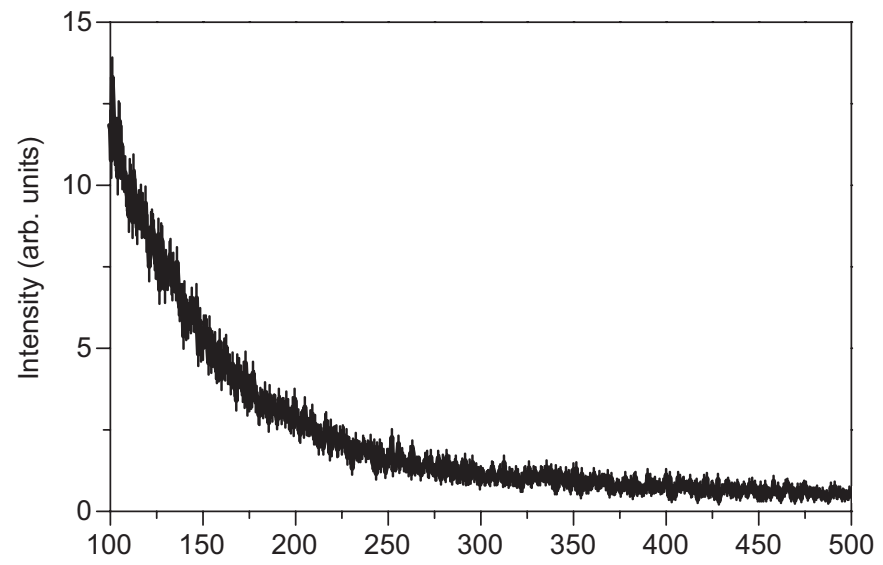

b

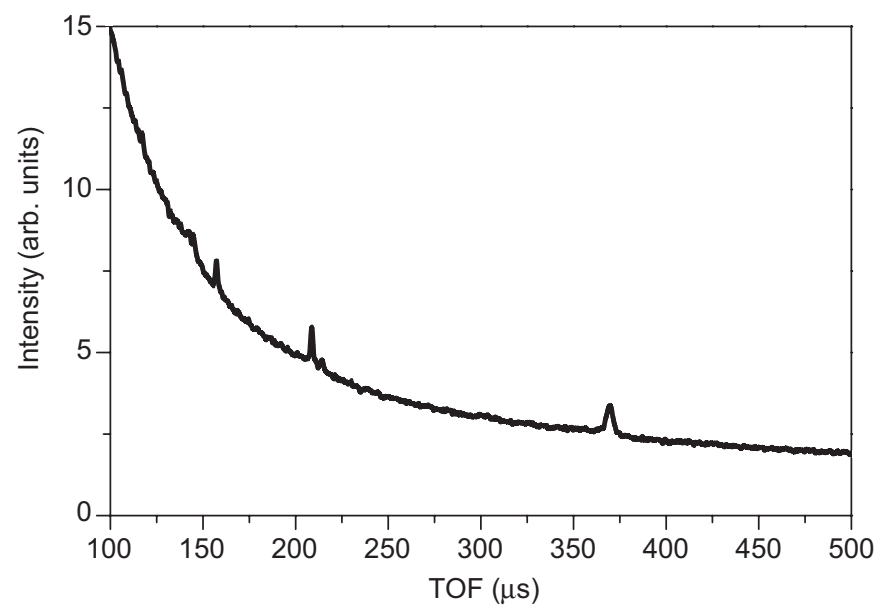

Fig. 6. (a) Time of flight spectrum without sample. (b) Time of flight spectrum with a $5 \mathrm{~mm}$ Perspex sample. The lack of statistically significant structures in the background spectrum (upper panel) is an indication of the transmitted beam not striking the detectors.

same ring can be added to simplify the analysis (see also Fig. 7).

\subsection{Signal and background assessment}

The VLAD detector units are sensitive to the gamma background of the VESUVIO experimental hall. Measure-
Table 1

Values of the calibrated instrumental parameters on VLAD

\begin{tabular}{llll}
\hline Det. ring & $L_{1}(\mathrm{~cm})$ & $2 \vartheta(\mathrm{deg})$ & $t_{0}(\mu \mathrm{s})$ \\
\hline 1 & $220 \pm 5$ & $0.96 \pm 1.00$ & $-1.3 \pm 0.8$ \\
2 & $206 \pm 5$ & $1.53 \pm 1.10$ & $-0.8 \pm 0.8$ \\
3 & $201 \pm 5$ & $2.08 \pm 1.20$ & $-0.8 \pm 0.8$ \\
4 & $198 \pm 5$ & $2.63 \pm 1.20$ & $-0.9 \pm 0.8$ \\
5 & $192 \pm 5$ & $3.34 \pm 1.50$ & $-0.7 \pm 0.8$ \\
\hline
\end{tabular}

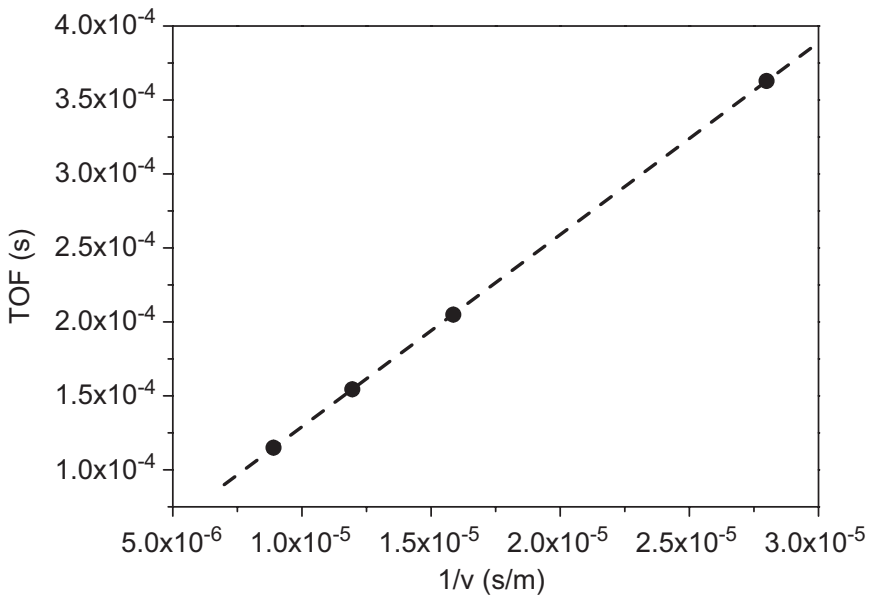

Fig. 7. Plot TOF versus inverse neutron velocity for the different $H$ elastic peaks recorded with the PERSPEX slab sample (see text for details). This fitting line is also shown: its slope is related to the total neutron flight path, while the intercept provides the time delay. The calibration of the scattering angle is done by using Eq. (5).

ments with a germanium detector have been conducted with the aim to characterize the gamma background on VESUVIO [32]. Recently, further tests have been conducted with YAP and YSO scintillators in order to better understand the nature of the background in the beamline, with the aim of finding suitable methods for its reduction, especially for the VLAD critical angles. The results obtained from these studies indicate the presence of different components of the gamma background: An important component at low time of flight resulted to be time-correlated with the neutron beam. Its origin is due to the gamma flash from the spallation process in the neutron 


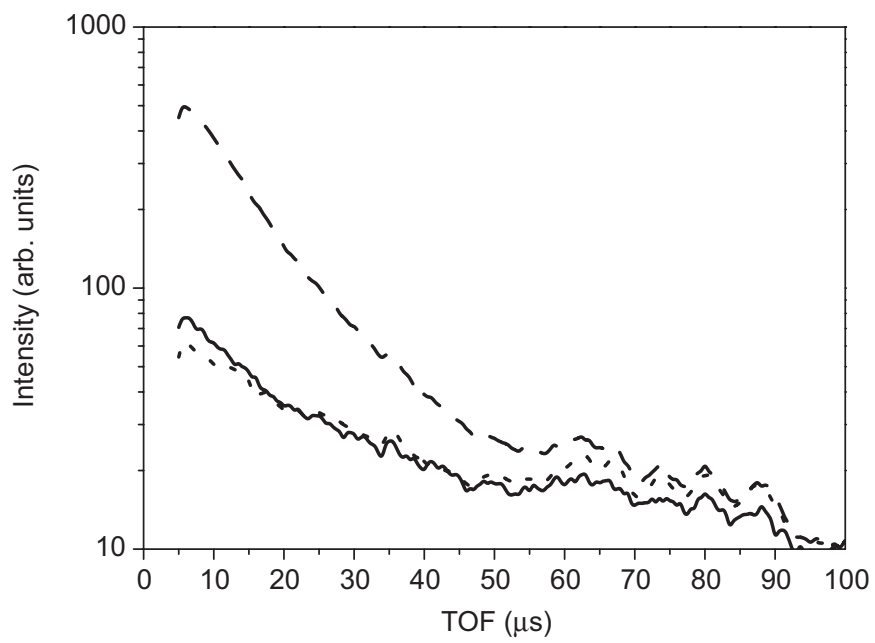

Fig. 8. Time of flight spectra acquired by a YSO detector enclosed in a lead shielding: the dashed line plot refers to a shielding configuration with an aperture towards the moderator, the continuous and dotted lines to apertures in opposite and at $90^{\circ}$ with respect to the moderator direction, respectively.

target and to radiative de-excitation after neutron capture in the incident beam pipe/collimation materials as well as from the moderator-decoupler-poisoning-liner system. Such a component is characterized by a strong directionality, as can be observed from Fig. 8. In this figure, TOF spectra acquired by a reference detector positioned close to the sample position and enclosed on a thick $(100 \mathrm{~mm}) 4 \pi$ lead shielding are shown. Measurements have been performed with partial shielding configurations, so as to allow background gamma from selected directions to reach the detector surface. A strong difference can be appreciated between the case of a shielding aperture directed towards the moderator and the other cases, especially at time of flight below $50 \mu \mathrm{s}$. This confirms that a strong gamma component comes from the target/moderator region and it is characterized by a high degree of directionality. The bumps present in all three spectra at about 60 and $80 \mu \mathrm{s}$ have been identified as due to $(n, \gamma)$ resonance reactions in $\mathrm{Sb}^{121}$ and $\mathrm{Sb}^{123}$ isotopes, present in the lead alloy of the detector shielding.

At longer time of flight the background level is almost the same for all cases, thus suggesting that the gamma background is mostly contributed by neutron scattering/ absorption in the walls of the experimental hall. This results in an almost isotropic contribution. Indeed, the main gamma background source at times longer than about $100 \mu$ s is the borated wax shielding [19]. Boron $\left({ }^{10} \mathrm{~B}\right)$ is commonly used in neutron shielding installations. Following a neutron capture event, ${ }^{10} \mathrm{~B}$ emits a prompt gamma at $477.6 \mathrm{keV}$, that may eventually be detected from the photon detectors, unless energy discrimination on the gamma-rays is introduced. Indeed, the TOF spectra in Fig. 8 have been acquired setting a lower level discrimination (LLD) threshold of about $600 \mathrm{keV}$, thus resulting in a complete electronic rejection of these photons. The use of an LLD was found to be essential in order to improve the signal to background ratio $(S / B)$ in the RD configuration $[19,33]$. Since the energy resolution of YAP scintillator is about $20 \%$ at $500 \mathrm{keV}$ [34], the LLD threshold level has to be chosen at about $600 \mathrm{keV}$ or higher in order to reject completely the ${ }^{10} \mathrm{~B}$ photons. As shown in Ref. [19], raising the threshold above this value does not provide significant variations.

A minor component of the background is due to the natural radioactivity of the uranium analyzer foils. This component, being due to random radioactive decays, contributes with a constant baseline in the TOF spectra. Its value can be estimated by interpolating the TOF spectrum at very long times (above $10 \mathrm{~ms}$ ), where the contribution of the neutron beam-related effects are practically negligible.

In order to reduce the directional gamma background for the VLAD detectors (especially for the ones placed in the innermost ring) two shielding systems were realized. They consist of two lead cylinders (with different contents of antimony) $7 \mathrm{~cm}$ diameter, $3 \mathrm{~mm}$ thickness and presenting a central hole of 35 and $25 \mathrm{~mm}$ diameter (identified as Lead_1 and Lead_2), respectively. These devices were inserted between the two sections of the incident beam

a

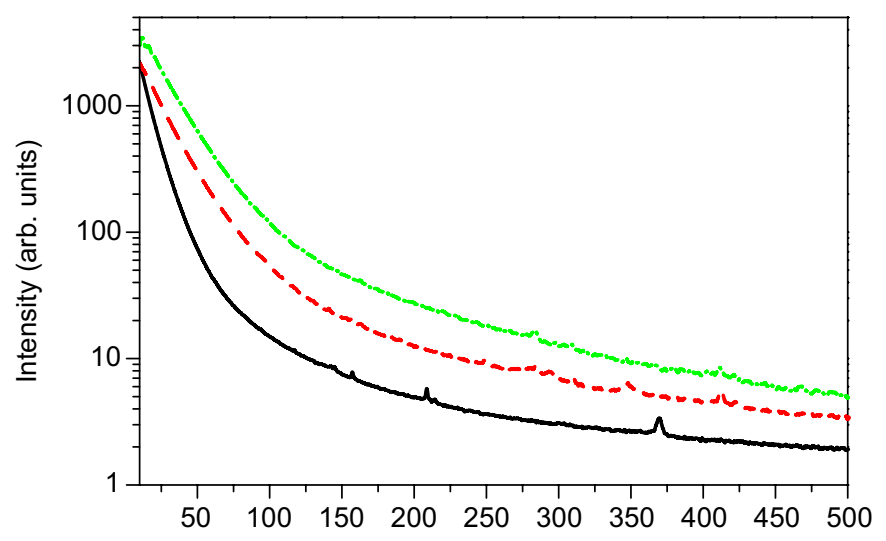

b

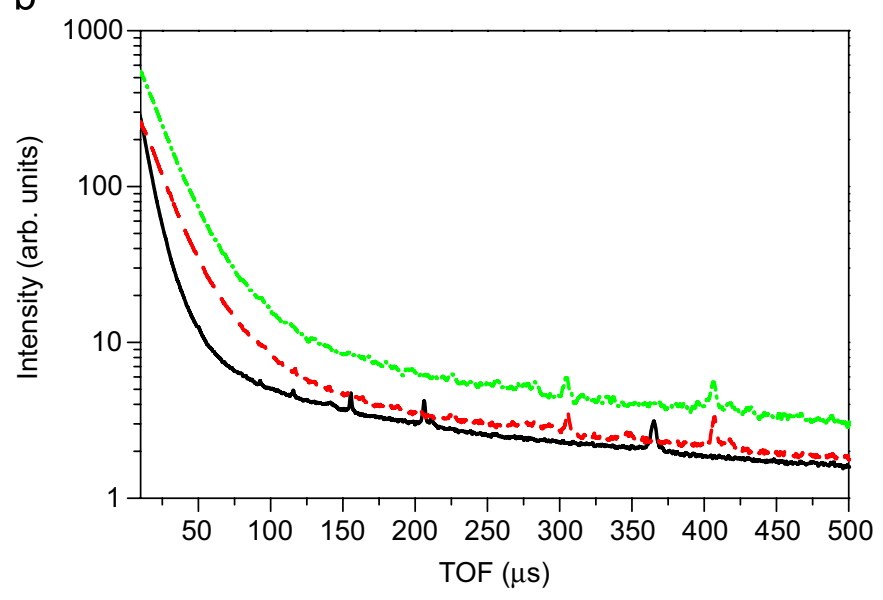

Fig. 9. Time of flight spectra from a Perspex sample recorded with Lead_1 (dashed line), Lead_2 (full line) and without lead insert (dashed-dotted line) for detectors at $1^{\circ}$ (a) and $4^{\circ}$ (b). 
pipe, as shown in Fig. 5. Fig. 9 shows TOF spectra acquired with different shieldings. The resonance peaks are visible in the TOF region around $80-100 \mu$ s (not to be confused with spurious peaks due to antimony in the second plot).

The improvement in the $S / B$ ratio due to the use of two different lead shielding elements can be appreciated as compared to the case where no shielding was used.

Calibrations with a vanadium sample are used in order to determine the non-uniform detector response. The normalized signal level is almost constant for all the detectors rings, with the exception of the innermost one. The latter shows that some detectors have a signal level about one half of the others belonging to the same ring. The reason for this lack of signal is possibly due to a possible detectors misalignment, together with a relatively higher background level as compared to the other rings.

\section{Conclusions}

The VLAD bank has been installed and successfully operated on the VESUVIO beam line at the ISIS spallation source. The vacuum tank and the detector array were aligned with respect to the transmitted neutron beam axis. The secondary flight path and the scattering angle of each detector of the array were calibrated and found to be in good agreement with construction specifications. Background and signal levels for the whole set of detectors were assessed. The background level is large and its reduction is a demanding task. The tests performed with lead shielding show that beam-related background can be significantly reduced. Future work will be devoted to reduce the samplerelated background.

In summary, VLAD provides an opportunity for new physics, as shown by the first measurements with benchmark samples, allowing for challenging but unique experimental investigations on condensed matter.

\section{Acknowledgments}

This work was supported within the CNR-CCLRC Agreement No. 01/9001 concerning collaboration in scientific research at the spallation neutron source ISIS. The financial support of the Consiglio Nazionale delle Ricerche in this research is hereby acknowledged. This work was performed with financial support by the European Community contract HPRI-2001-50043.

The authors greatly acknowledge R. Alessandroni and F. Lama (RMP-Acilia, Rome) and B. Holsman (ISIS facility) for collaboration in the design stage and for the construction of the VLAD vacuum tank and detectors frame.

\section{References}

[1] C. Andreani, D. Colognesi, J. Mayers, G.F. Reiter, R. Senesi, Adv. Phys. 54 (2006) 377.
[2] S.W. Lovesey, Theory of Neutron Scattering from Condensed Matter, third ed., Oxford University Press, Oxford, 1987.

[3] R.O. Simmons, Z. Naturforsch. 48a (1993) 415.

[4] C. Andreani, G. Gorini, E. Perelli-Cippo, A. Pietropaolo, N.J. Rhodes, E.M. Schooneveld, R. Senesi, M. Tardocchi, Appl. Phys. Lett. 85 (2004) 5454.

[5] C. Andreani, A. Pietropaolo, R. Senesi, G. Gorini, M. Tardocchi, N. Rhodes, E.M. Schooneveld, A. D' Angelo, Condensed matter studies with $20-100 \mathrm{eV}$ neutrons: effective detection systems for high inelastic neutron scattering and deep inelastic neutron scattering, in: ISIS2003 Science Highlights.

[6] D. Richter, in: Proceedings of the 1984 Workshop on High Energy Excitations in Condensed Matter, Los Alamos, New Mexico, Febraury 13-15, 1984. p. 559; J. Eckert, in: Proceedings of the 1984 Workshop on High Energy Excitations in Condensed Matter, Los Alamos, New Mexico, Febraury 13-15, 1984, p. 540.

[7] J. Howard, in: Proceedings of the 1984 Workshop on High Energy Excitations in Condensed Matter, Los Alamos, New Mexico, Febraury 13-15, 1984, p. 548; P.A. Egelstaff, in: Proceedings of the 1984 Workshop on High Energy Excitations in Condensed Matter, Los Alamos, New Mexico, Febraury 13-15, 1984, p. 626 and references therein.

[8] H.A. Mook, in: Proceedings of the 1984 Workshop on High Energy Excitations in Condensed Matter, Los Alamos, New Mexico, Febraury 13-15, 1984, p. 226; M. Loewenhaupt, in: Proceedings of the 1984 Workshop on High Energy Excitations in Condensed Matter, Los Alamos, New Mexico, Febraury 13-15, 1984, p. 315 and references therein.

[9] J.F. Cook, J.A. Blackman, T. Morgan, in: Proceedings of the 1984 Workshop on High Energy Excitations in Condensed Matter, Los Alamos, New Mexico, Febraury 13-15, 1984, p. 401 and reference therein.

[10] A. Pietropaolo, R. Senesi, M. Tardocchi, C. Andreani, G. Gorini, Physica B 350 (2004) e857.

[11] A.D. Taylor, R.A. Robinson, P.A. Seeger, Nucl. Instr. and Meth. 224 (1984) 133

[12] S.K. Sinha, J. Appl. Phys. 50 (3) (1979) 1952.

[13] H. Rauh, N. Watanabe, Nucl. Instr. and Meth. 228 (1984) 147; N. Watanabe, in: Proceedings of the 1984 Workshop on High Energy Excitations in Condensed Matter, Los Alamos, February 13-15, LA-10227-C, vol. 1, 1984;

J.M. Carpenter, N. Watanabe, S. Ikeda, Y. Masuda, S. Sato, H. Rauh, Physica B 120 (1983) 126.

[14] R.N. Sinclair, M.C. Moxon, J.M. Carpenter, Bull. Am. Phys. Soc. 22 (1977) 101 ;

L. Cser, N. Kroó, P. Pacher, V.G. Simkin, E.V. Vasilieva, Nucl. Instr. and Meth. 179 (1981) 515;

D.R. Allen, E.W.J. Mitchell, R.N. Sinclair, J. Phys. E 13 (1983) 639

[15] C. Andreani, A. Pietropaolo, R. Senesi, G. Gorini, M. Tardocchi, A. Bracco, N. Rhodes, E. Schooneveld, Nucl. Instr. and Meth. A 481 (2002) 509.

[16] A. Pietropaolo, C. Andreani, A. D'Angelo, G. Gorini, S. Imberti, M. Tardocchi, N. Rhodes, E.M. Schooneveld, R. Senesi, in: Proceedings of the 11th International Symposium on Capture Gamma-Ray spectroscopy and related Topics, vol. 555, World Scientific, Singapore, 555, 2003.

[17] 〈www.isis.rl.ac.uk〉.

[18] M. Tardocchi, A. Pietropaolo, C. Andreani, A. Bracco, A. D’Angelo, G. Gorini, S. Imberti, R. Senesi, N.J. Rhodes, E.M. Schooneveld, Nucl. Instr. and Meth. A 526 (2004) 477.

[19] M. Tardocchi, G. Gorini, A. Pietropaolo, C. Andreani, R. Senesi, N. Rhodes, E.M. Schooneveld, Rev. Sci. Instr. 75 (2004) 4880.

[20] A. Pietropaolo, C. Andreani, A. Filabozzi, G. Gorini, N. Rhodes, R. Senesi, E. Schooneveld, M. Tardocchi, JINST 1 P04001, doi: 10.1088/1748-0221/1/04/P04001.

[21] C. Andreani, P. Bosi, F. Sacchetti, C.K. Loong, J. Chem. Phys. 83 (1985) 750.

[22] J.S. Scwinger, Phys. Rev. 51 (1937) 544. 
[23] G.T. Trammel, Phys. Rev. 92 (1953) 1387.

[24] E. Balcar, S.W. Lovesey, J. Phys. C 19 (1986) 4605.

[25] C. Stassis, H.W. Deckman, Phys. Rev. B 12 (1975) 1885.

[26] L.L. Foldy, Phys. Rev. 87 (1952) 693.

[27] A.D. Taylor, R. Osborn, K.A. McEwen, W.G. Stirling, Z.A. Bowden, W.G. Williams, E. Balcar, S.W. Lovesy, Phys. Rev. Lett. 61 (1988) 1309.

[28] C.G. Windsor, Pulsed Neutron Scattering, Taylor \& Francis, Bristol, PA, 1981.

[29] 〈http://www.fisica.uniroma2.it/vesuvio/dins/index.html $\rangle$.
[30] Agostinelli, et al., Nucl. Instr. and Meth. A 506 (2003) 250.

[31] E. Perelli-Cippo, G. Gorini, O. Cremonesi, M. Tadocchi, C. Andreani, A. Pietropaolo, R. Senesi, N.J. Rhodes, E.M. Schooneveld, IEEE Trans. Nucl. Sci. 52 (2005) 1092.

[32] A. Pietropaolo, M. Tardocchi, E.M. Schooneveld, R. Senesi, Nucl. Instr. and Meth. A 568 (2006) 826.

[33] M. Tardocchi, A. Pietropaolo, C. Andreani, G. Gorini, E. Perelli-Cippo, N.J. Rhodes, E.M. Schooneveld, Nucl. Instr. and Meth. A 535 (2004) 121.

[34] 〈www.detectors.saint-gobain.com〉 (data compiled by C. Rozsa). 